\title{
Anhang 2b: Follower-Netzwerk (Alternative und professionelle Nachrichtenmedien)
}

\begin{tabular}{|c|c|c|c|c|c|c|c|}
\hline Account & $\begin{array}{l}\text { Modularity } \\
\text { Class }\end{array}$ & $\begin{array}{c}\text { In- } \\
\text { degree }\end{array}$ & $\begin{array}{c}\text { Out- } \\
\text { degree }\end{array}$ & Degree & $\begin{array}{c}\text { Close- } \\
\text { ness } \\
\text { Centrality }\end{array}$ & $\begin{array}{l}\text { Betweenness } \\
\text { Centrality }\end{array}$ & $\begin{array}{l}\text { Eigen. } \\
\text { Centra- } \\
\text { lity }\end{array}$ \\
\hline $\begin{array}{l}\text { Natascha_ } \\
\text { Strobl }\end{array}$ & $\begin{array}{l}\text { Österreich- } \\
\text { Community }\end{array}$ & 11796 & 2050 & 13846 & 0,365 & 114866482,828 & 0,443 \\
\hline ArminWolf & $\begin{array}{l}\text { Österreich- } \\
\text { Community }\end{array}$ & 11580 & 363 & 11943 & 0,315 & 15175592,767 & 0,491 \\
\hline sebastiankurz & $\begin{array}{l}\text { Österreich- } \\
\text { Community }\end{array}$ & 9635 & 287 & 9922 & 0,310 & 17909182,955 & 0,380 \\
\hline florianklenk & $\begin{array}{l}\text { Österreich- } \\
\text { Community }\end{array}$ & 8775 & 627 & 9402 & 0,329 & 16071331,616 & 0,391 \\
\hline vanderbellen & $\begin{array}{l}\text { Österreich- } \\
\text { Community }\end{array}$ & 6820 & 116 & 6936 & 0,299 & 3094669,858 & 0,258 \\
\hline derStandardat & $\begin{array}{l}\text { Österreich- } \\
\text { Community }\end{array}$ & 6381 & 295 & 6676 & 0,333 & 40428966,255 & 0,251 \\
\hline corinnamilborn & $\begin{array}{l}\text { Österreich- } \\
\text { Community }\end{array}$ & 5454 & 714 & 6168 & 0,339 & 11533569,043 & 0,260 \\
\hline misik & $\begin{array}{l}\text { Österreich- } \\
\text { Community }\end{array}$ & 4891 & 1555 & 6446 & 0,356 & 25875505,013 & 0,262 \\
\hline brodnig & $\begin{array}{l}\text { Österreich- } \\
\text { Community }\end{array}$ & 4798 & 723 & 5521 & 0,334 & 6106865,850 & 0,256 \\
\hline KernChri & $\begin{array}{l}\text { Österreich- } \\
\text { Community }\end{array}$ & 4558 & 46 & 4604 & 0,290 & 580309,916 & 0,193 \\
\hline falter_at & $\begin{array}{l}\text { Österreich- } \\
\text { Community }\end{array}$ & 4322 & 45 & 4367 & 0,286 & 202841,676 & 0,162 \\
\hline IngridThurnher & $\begin{array}{l}\text { Österreich- } \\
\text { Community }\end{array}$ & 4092 & 515 & 4607 & 0,325 & 4479856,552 & 0,172 \\
\hline WKogler & $\begin{array}{l}\text { Österreich- } \\
\text { Community }\end{array}$ & 4056 & 1020 & 5076 & 0,338 & 10629120,074 & 0,169 \\
\hline profilonline & $\begin{array}{l}\text { Österreich- } \\
\text { Community }\end{array}$ & 3867 & 502 & 4369 & 0,331 & 5475784,800 & 0,155 \\
\hline KURIERat & $\begin{array}{l}\text { Österreich- } \\
\text { Community }\end{array}$ & 3644 & 1221 & 4865 & 0,349 & 20221302,107 & 0,147 \\
\hline DiePressecom & $\begin{array}{l}\text { Österreich- } \\
\text { Community }\end{array}$ & 3435 & 488 & 3923 & 0,328 & 10698182,059 & 0,132 \\
\hline
\end{tabular}




\begin{tabular}{|c|c|c|c|c|c|c|c|}
\hline Account & $\begin{array}{l}\text { Modularity } \\
\text { Class }\end{array}$ & $\begin{array}{c}\text { In- } \\
\text { degree }\end{array}$ & $\begin{array}{c}\text { Out- } \\
\text { degree }\end{array}$ & Degree & $\begin{array}{l}\text { Close- } \\
\text { ness } \\
\text { Centrality }\end{array}$ & $\begin{array}{l}\text { Betweenness } \\
\text { Centrality }\end{array}$ & $\begin{array}{l}\text { Eigen. } \\
\text { Centra- } \\
\text { lity }\end{array}$ \\
\hline Gruene_Austria & $\begin{array}{l}\text { Österreich- } \\
\text { Community }\end{array}$ & 3270 & 467 & 3737 & 0,319 & 2502973,673 & 0,139 \\
\hline BMeinl & $\begin{array}{l}\text { Österreich- } \\
\text { Community }\end{array}$ & 3221 & 977 & 4198 & 0,342 & 10875352,531 & 0,141 \\
\hline rendiwagner & $\begin{array}{l}\text { Österreich- } \\
\text { Community }\end{array}$ & 3002 & 122 & 3124 & 0,293 & 274024,053 & 0,124 \\
\hline daswasfehlt & $\begin{array}{l}\text { Österreich- } \\
\text { Community }\end{array}$ & 2904 & 69 & 2973 & 0,299 & 506086,700 & 0,128 \\
\hline WienerZeitung & $\begin{array}{l}\text { Österreich- } \\
\text { Community }\end{array}$ & 2874 & 538 & 3412 & 0,327 & 3916696,185 & 0,122 \\
\hline SPOE_at & $\begin{array}{l}\text { Österreich- } \\
\text { Community }\end{array}$ & 2763 & 366 & 3129 & 0,315 & 1970379,503 & 0,112 \\
\hline Kontrast_at & $\begin{array}{l}\text { Österreich- } \\
\text { Community }\end{array}$ & 2738 & 20 & 2758 & 0,284 & 113307,312 & 0,103 \\
\hline neos_eu & $\begin{array}{l}\text { Österreich-Com- } \\
\text { munity }\end{array}$ & 2708 & 282 & 2990 & 0,313 & 1463560,606 & 0,114 \\
\hline webstandardat & $\begin{array}{l}\text { Österreich- } \\
\text { Community }\end{array}$ & 2630 & 753 & 3383 & 0,353 & 24762698,450 & 0,100 \\
\hline krone_at & $\begin{array}{l}\text { Österreich- } \\
\text { Community }\end{array}$ & 2630 & 21 & 2651 & 0,282 & 795557,348 & 0,102 \\
\hline futurezoneat & $\begin{array}{l}\text { Österreich- } \\
\text { Community }\end{array}$ & 2484 & 738 & 3222 & 0,340 & 9364942,796 & 0,105 \\
\hline volkspartei & $\begin{array}{l}\text { Österreich-Com- } \\
\text { munity }\end{array}$ & 2099 & 387 & 2486 & 0,315 & 1675552,768 & 0,088 \\
\hline ORF & $\begin{array}{l}\text { Österreich- } \\
\text { Community }\end{array}$ & 2097 & 0 & 2097 & 0,000 & 0,000 & 0,085 \\
\hline mosaik_blog & $\begin{array}{l}\text { Österreich- } \\
\text { Community }\end{array}$ & 1252 & 128 & 1380 & 0,299 & 184216,036 & 0,064 \\
\hline zeitonline & $\begin{array}{l}\text { Deutschland- } \\
\text { Community }\end{array}$ & 28842 & 26 & 28868 & 0,281 & 4487305,638 & 1,000 \\
\hline janboehm & $\begin{array}{l}\text { Deutschland- } \\
\text { Community }\end{array}$ & 28388 & 545 & 28933 & 0,335 & 126159815,426 & 0,938 \\
\hline tagesschau & $\begin{array}{l}\text { Deutschland- } \\
\text { Community }\end{array}$ & 27958 & 159 & 28117 & 0,297 & 2526495,002 & 0,926 \\
\hline GretaThunberg & $\begin{array}{l}\text { Deutschland- } \\
\text { Community }\end{array}$ & 27433 & 123 & 27556 & 0,316 & 282503514,616 & 0,821 \\
\hline SPIEGELONLINE & $\begin{array}{l}\text { Deutschland- } \\
\text { Community }\end{array}$ & 27099 & 128 & 27227 & 0,304 & 33785627,627 & 0,922 \\
\hline SZ & $\begin{array}{l}\text { Deutschland- } \\
\text { Community }\end{array}$ & 25930 & 181 & 26111 & 0,302 & 22022281,335 & 0,904 \\
\hline Der_Postillon & $\begin{array}{l}\text { Deutschland- } \\
\text { Community }\end{array}$ & 23114 & 89 & 23203 & 0,314 & 30293924,028 & 0,712 \\
\hline
\end{tabular}




\begin{tabular}{|c|c|c|c|c|c|c|c|}
\hline Account & $\begin{array}{l}\text { Modularity } \\
\text { Class }\end{array}$ & $\begin{array}{c}\text { In- } \\
\text { degree }\end{array}$ & $\begin{array}{c}\text { Out- } \\
\text { degree }\end{array}$ & Degree & $\begin{array}{c}\text { Close- } \\
\text { ness } \\
\text { Centrality }\end{array}$ & $\begin{array}{c}\text { Betweenness } \\
\text { Centrality }\end{array}$ & $\begin{array}{c}\text { Eigen. } \\
\text { Centra- } \\
\text { lity }\end{array}$ \\
\hline tazgezwitscher & $\begin{array}{l}\text { Deutschland- } \\
\text { Community }\end{array}$ & 20144 & 578 & 20722 & 0,336 & 43429522,112 & 0,773 \\
\hline welt & $\begin{array}{l}\text { Deutschland- } \\
\text { Community }\end{array}$ & 17923 & 69 & 17992 & 0,300 & 12669525,196 & 0,636 \\
\hline SPIEGEL_EIL & $\begin{array}{l}\text { Deutschland- } \\
\text { Community }\end{array}$ & 17717 & 11 & 17728 & 0,238 & 1002473,368 & 0,645 \\
\hline faznet & $\begin{array}{l}\text { Deutschland- } \\
\text { Community }\end{array}$ & 16895 & 181 & 17076 & 0,307 & 14408259,105 & 0,615 \\
\hline ZDF & $\begin{array}{l}\text { Deutschland- } \\
\text { Community }\end{array}$ & 16354 & 169 & 16523 & 0,313 & 17722932,672 & 0,595 \\
\hline dpa & $\begin{array}{l}\text { Deutschland- } \\
\text { Community }\end{array}$ & 16037 & 720 & 16757 & 0,334 & 42840138,810 & 0,638 \\
\hline ZDFheute & $\begin{array}{l}\text { Deutschland- } \\
\text { Community }\end{array}$ & 15057 & 135 & 15192 & 0,311 & 13757726,926 & 0,542 \\
\hline titanic & $\begin{array}{l}\text { Deutschland- } \\
\text { Community }\end{array}$ & 14708 & 8 & 14716 & 0,258 & 606888,729 & 0,517 \\
\hline Tagesspiegel & $\begin{array}{l}\text { Deutschland- } \\
\text { Community }\end{array}$ & 13323 & 710 & 14033 & 0,341 & 34015764,179 & 0,555 \\
\hline Die_Gruenen & $\begin{array}{l}\text { Deutschland- } \\
\text { Community }\end{array}$ & 13275 & 1563 & 14838 & 0,361 & 90448912,106 & 0,506 \\
\hline DerSPIEGEL & $\begin{array}{l}\text { Deutschland- } \\
\text { Community }\end{array}$ & 13140 & 122 & 13262 & 0,300 & 10949795,635 & 0,499 \\
\hline neomagazin & $\begin{array}{l}\text { Deutschland- } \\
\text { Community }\end{array}$ & 13075 & 1 & 13076 & 0,251 & 166092,452 & 0,424 \\
\hline BILDblog & $\begin{array}{l}\text { Deutschland- } \\
\text { Community }\end{array}$ & 12866 & 67 & 12933 & 0,296 & 2877683,726 & 0,512 \\
\hline DIEZEIT & $\begin{array}{l}\text { Deutschland- } \\
\text { Community }\end{array}$ & 12549 & 578 & 13127 & 0,331 & 26100597,237 & 0,507 \\
\hline sternde & $\begin{array}{l}\text { Deutschland- } \\
\text { Community }\end{array}$ & 12304 & 205 & 12509 & 0,319 & 23528411,810 & 0,433 \\
\hline ntvde & $\begin{array}{l}\text { Deutschland- } \\
\text { Community }\end{array}$ & 11395 & 2069 & 13464 & 0,387 & 428257628,778 & 0,432 \\
\hline DasErste & $\begin{array}{l}\text { Deutschland- } \\
\text { Community }\end{array}$ & 11081 & 146 & 11227 & 0,296 & 2951847,405 & 0,416 \\
\hline BILD & $\begin{array}{l}\text { Deutschland- } \\
\text { Community }\end{array}$ & 11036 & 196 & 11232 & 0,302 & 22052464,996 & 0,409 \\
\hline rezomusik & $\begin{array}{l}\text { Deutschland- } \\
\text { Community }\end{array}$ & 10899 & 159 & 11058 & 0,315 & 29289197,547 & 0,361 \\
\hline FridayForFuture & $\begin{array}{l}\text { Deutschland- } \\
\text { Community }\end{array}$ & 10627 & 0 & 10627 & 0,000 & 0,000 & 0,342 \\
\hline correctiv_org & $\begin{array}{l}\text { Deutschland- } \\
\text { Community }\end{array}$ & 10338 & 1582 & 11920 & 0,368 & 79043439,219 & 0,493 \\
\hline
\end{tabular}




\begin{tabular}{|c|c|c|c|c|c|c|c|}
\hline Account & $\begin{array}{l}\text { Modularity } \\
\text { Class }\end{array}$ & $\begin{array}{c}\text { In- } \\
\text { degree }\end{array}$ & $\begin{array}{l}\text { Out- } \\
\text { degree }\end{array}$ & Degree & $\begin{array}{l}\text { Close- } \\
\text { ness } \\
\text { Centrality }\end{array}$ & $\begin{array}{l}\text { Betweenness } \\
\text { Centrality }\end{array}$ & $\begin{array}{l}\text { Eigen. } \\
\text { Centra- } \\
\text { lity }\end{array}$ \\
\hline DiePARTEI & $\begin{array}{l}\text { Deutschland- } \\
\text { Community }\end{array}$ & 10107 & 89 & 10196 & 0,304 & 11963379,928 & 0,290 \\
\hline spdde & $\begin{array}{l}\text { Deutschland- } \\
\text { Community }\end{array}$ & 9587 & 1369 & 10956 & 0,360 & 55217402,640 & 0,408 \\
\hline WDR & $\begin{array}{l}\text { Deutschland- } \\
\text { Community }\end{array}$ & 9316 & 320 & 9636 & 0,316 & 9688146,961 & 0,359 \\
\hline derfreitag & $\begin{array}{l}\text { Deutschland- } \\
\text { Community }\end{array}$ & 9284 & 1422 & 10706 & 0,363 & 62387104,440 & 0,434 \\
\hline dieLinke & $\begin{array}{l}\text { Deutschland- } \\
\text { Community }\end{array}$ & 9275 & 672 & 9947 & 0,341 & 29374114,317 & 0,346 \\
\hline tagesschau_eil & $\begin{array}{l}\text { Deutschland- } \\
\text { Community }\end{array}$ & 9234 & 1 & 9235 & 0,229 & 132745,580 & 0,364 \\
\hline bundesrat & $\begin{array}{l}\text { Deutschland- } \\
\text { Community }\end{array}$ & 8993 & 343 & 9336 & 0,318 & 6863558,528 & 0,350 \\
\hline greenpeace_de & $\begin{array}{l}\text { Deutschland- } \\
\text { Community }\end{array}$ & 8814 & 400 & 9214 & 0,333 & 18268253,971 & 0,315 \\
\hline $\begin{array}{l}\text { GrueneBun- } \\
\text { destag }\end{array}$ & $\begin{array}{l}\text { Deutschland- } \\
\text { Community }\end{array}$ & 8328 & 315 & 8643 & 0,327 & 5868879,447 & 0,352 \\
\hline tagesthemen & $\begin{array}{l}\text { Deutschland- } \\
\text { Community }\end{array}$ & 8274 & 261 & 8535 & 0,309 & 3582903,311 & 0,352 \\
\hline Volksverpetzer & $\begin{array}{l}\text { Deutschland- } \\
\text { Community }\end{array}$ & 8201 & 287 & 8488 & 0,332 & 13177984,470 & 0,267 \\
\hline CDU & $\begin{array}{l}\text { Deutschland- } \\
\text { Community }\end{array}$ & 8195 & 552 & 8747 & 0,331 & 14763258,524 & 0,347 \\
\hline krautreporter & $\begin{array}{l}\text { Deutsch- } \\
\text { land-Community }\end{array}$ & 7761 & 795 & 8556 & 0,352 & 30392137,415 & 0,366 \\
\hline phoenix_de & $\begin{array}{l}\text { Deutschland- } \\
\text { Community }\end{array}$ & 7677 & 464 & 8141 & 0,326 & 6373839,236 & 0,346 \\
\hline uebermedien & $\begin{array}{l}\text { Deutschland- } \\
\text { Community }\end{array}$ & 7612 & 109 & 7721 & 0,310 & 1467792,488 & 0,361 \\
\hline NZZ & $\begin{array}{l}\text { Schweiz- } \\
\text { Community }\end{array}$ & 15732 & 1417 & 17149 & 0,358 & 201042302,360 & 0,576 \\
\hline srfnews & $\begin{array}{l}\text { Schweiz- } \\
\text { Community }\end{array}$ & 6116 & 31 & 6147 & 0,276 & 2189143,277 & 0,199 \\
\hline tagesanzeiger & $\begin{array}{l}\text { Schweiz- } \\
\text { Community }\end{array}$ & 5832 & 733 & 6565 & 0,335 & 25245752,400 & 0,204 \\
\hline $\begin{array}{l}\text { RepublikMa- } \\
\text { gazin }\end{array}$ & $\begin{array}{l}\text { Schweiz- } \\
\text { Community }\end{array}$ & 4934 & 71 & 5005 & 0,304 & 1633064,699 & 0,215 \\
\hline watson_news & $\begin{array}{l}\text { Schweiz- } \\
\text { Community }\end{array}$ & 4860 & 1708 & 6568 & 0,369 & 81270193,300 & 0,185 \\
\hline SRF & $\begin{array}{l}\text { Schweiz- } \\
\text { Community }\end{array}$ & 4280 & 131 & 4411 & 0,298 & 2547611,007 & 0,150 \\
\hline
\end{tabular}




\begin{tabular}{|c|c|c|c|c|c|c|c|}
\hline Account & $\begin{array}{l}\text { Modularity } \\
\text { Class }\end{array}$ & $\begin{array}{c}\text { In- } \\
\text { degree }\end{array}$ & $\begin{array}{c}\text { Out- } \\
\text { degree }\end{array}$ & Degree & $\begin{array}{c}\text { Close- } \\
\text { ness } \\
\text { Centrality }\end{array}$ & $\begin{array}{c}\text { Betweenness } \\
\text { Centrality }\end{array}$ & $\begin{array}{c}\text { Eigen. } \\
\text { Centra- } \\
\text { lity }\end{array}$ \\
\hline Blickch & $\begin{array}{l}\text { Schweiz- } \\
\text { Community }\end{array}$ & 3813 & 417 & 4230 & 0,321 & 13049068,996 & 0,133 \\
\hline FabianEberhard & $\begin{array}{l}\text { Schweiz- } \\
\text { Community }\end{array}$ & 3769 & 470 & 4239 & 0,330 & 13952838,657 & 0,206 \\
\hline Wochenzeitung & $\begin{array}{l}\text { Schweiz- } \\
\text { Community }\end{array}$ & 3672 & 24 & 3696 & 0,275 & 219368,059 & 0,150 \\
\hline Weltwoche & $\begin{array}{l}\text { Schweiz- } \\
\text { Community }\end{array}$ & 3344 & 337 & 3681 & 0,334 & 12966114,288 & 0,130 \\
\hline NZZaS & $\begin{array}{l}\text { Schweiz- } \\
\text { Community }\end{array}$ & 3244 & 87 & 3331 & 0,295 & 846064,920 & 0,130 \\
\hline adfichter & $\begin{array}{l}\text { Schweiz- } \\
\text { Community }\end{array}$ & 3244 & 1061 & 4305 & 0,353 & 13432862,262 & 0,172 \\
\hline $\begin{array}{l}\text { sonntags } \\
\text { zeitung }\end{array}$ & $\begin{array}{l}\text { Schweiz- } \\
\text { Community }\end{array}$ & 3011 & 26 & 3037 & 0,280 & 164048,262 & 0,115 \\
\hline BR_Sprecher & $\begin{array}{l}\text { Schweiz- } \\
\text { Community }\end{array}$ & 2950 & 30 & 2980 & 0,256 & 384762,477 & 0,110 \\
\hline bazonline & $\begin{array}{l}\text { Schweiz- } \\
\text { Community }\end{array}$ & 2839 & 116 & 2955 & 0,288 & 2247608,982 & 0,109 \\
\hline spschweiz & $\begin{array}{l}\text { Schweiz- } \\
\text { Community }\end{array}$ & 2628 & 560 & 3188 & 0,324 & 2972438,661 & 0,097 \\
\hline srf3 & $\begin{array}{l}\text { Schweiz- } \\
\text { Community }\end{array}$ & 2572 & 115 & 2687 & 0,296 & 1636015,081 & 0,086 \\
\hline persoenlichcom & $\begin{array}{l}\text { Schweiz-Com- } \\
\text { munity }\end{array}$ & 2461 & 297 & 2758 & 0,312 & 1364114,748 & 0,103 \\
\hline GrueneCH & $\begin{array}{l}\text { Schweiz- } \\
\text { Community }\end{array}$ & 2427 & 593 & 3020 & 0,326 & 5251229,116 & 0,096 \\
\hline FDP_Liberalen & $\begin{array}{l}\text { Schweiz- } \\
\text { Community }\end{array}$ & 2314 & 932 & 3246 & 0,333 & 8968627,901 & 0,092 \\
\hline grunliberale & $\begin{array}{l}\text { Schweiz- } \\
\text { Community }\end{array}$ & 2166 & 409 & 2575 & 0,317 & 2550035,167 & 0,082 \\
\hline Medienwoche & $\begin{array}{l}\text { Schweiz- } \\
\text { Community }\end{array}$ & 2089 & 382 & 2471 & 0,315 & 1233834,631 & 0,100 \\
\hline RolandTichy & $\begin{array}{l}\text { Alternativme- } \\
\text { dien Typ I, II }\end{array}$ & 8801 & 338 & 9139 & 0,337 & 18914883,540 & 0,354 \\
\hline cicero_online & $\begin{array}{l}\text { Alternativme- } \\
\text { dien Typ I, II }\end{array}$ & 7057 & 562 & 7619 & 0,349 & 24632970,124 & 0,318 \\
\hline Alice_Weidel & $\begin{array}{l}\text { Alternativme- } \\
\text { dien Typ I, II }\end{array}$ & 7040 & 256 & 7296 & 0,320 & 12860730,912 & 0,261 \\
\hline AfD & $\begin{array}{l}\text { Alternativme- } \\
\text { dien Typ I, II }\end{array}$ & 6254 & 356 & 6610 & 0,327 & 19677143,350 & 0,236 \\
\hline Steinhoefel & $\begin{array}{l}\text { Alternativme- } \\
\text { dien Typ I, II }\end{array}$ & 6244 & 75 & 6319 & 0,305 & 1386014,734 & 0,234 \\
\hline
\end{tabular}




\begin{tabular}{|c|c|c|c|c|c|c|c|}
\hline Account & $\begin{array}{l}\text { Modularity } \\
\text { Class }\end{array}$ & $\begin{array}{c}\text { In- } \\
\text { degree }\end{array}$ & $\begin{array}{c}\text { Out- } \\
\text { degree }\end{array}$ & Degree & $\begin{array}{c}\text { Close- } \\
\text { ness } \\
\text { Centrality } \\
\end{array}$ & $\begin{array}{c}\text { Betweenness } \\
\text { Centrality }\end{array}$ & $\begin{array}{c}\text { Eigen. } \\
\text { Centra- } \\
\text { lity } \\
\end{array}$ \\
\hline TichysEinblick & $\begin{array}{l}\text { Alternativme- } \\
\text { dien Typ I, II }\end{array}$ & 6152 & 64 & 6216 & 0,305 & 2551905,007 & 0,216 \\
\hline Achgut_com & $\begin{array}{l}\text { Alternativme- } \\
\text { dien Typ I, II }\end{array}$ & 5664 & 168 & 5832 & 0,320 & 4494192,468 & 0,210 \\
\hline Junge_Freiheit & $\begin{array}{l}\text { Alternativme- } \\
\text { dien Typ I, II }\end{array}$ & 5008 & 302 & 5310 & 0,330 & 6962531,180 & 0,191 \\
\hline $\begin{array}{l}\text { AfDimBundes- } \\
\text { tag }\end{array}$ & $\begin{array}{l}\text { Alternativme- } \\
\text { dien Typ I, II }\end{array}$ & 4540 & 234 & 4774 & 0,312 & 3772103,511 & 0,181 \\
\hline RT_Deutsch & $\begin{array}{l}\text { Alternativme- } \\
\text { dien Typ I, II }\end{array}$ & 4268 & 94 & 4362 & 0,313 & 4729387,764 & 0,152 \\
\hline Hartes_Geld & $\begin{array}{l}\text { Alternativme- } \\
\text { dien Typ I, II }\end{array}$ & 4243 & 276 & 4519 & 0,326 & 5970343,560 & 0,158 \\
\hline KoeppelRoger & $\begin{array}{l}\text { Alternativme- } \\
\text { dien Typ I, II }\end{array}$ & 4178 & 55 & 4233 & 0,300 & 1848061,603 & 0,167 \\
\hline $\begin{array}{l}\text { COMPACT } \\
\text { Magazin }\end{array}$ & $\begin{array}{l}\text { Alternativme- } \\
\text { dien Typ I, II }\end{array}$ & 3840 & 992 & 4832 & 0,348 & 31499418,932 & 0,151 \\
\hline $\begin{array}{l}\text { NachDenk- } \\
\text { Seiten }\end{array}$ & $\begin{array}{l}\text { Alternativme- } \\
\text { dien Typ I, II }\end{array}$ & 3831 & 41 & 3872 & 0,301 & 717942,172 & 0,138 \\
\hline BjoernHoecke & $\begin{array}{l}\text { Alternativme- } \\
\text { dien Typ I, II }\end{array}$ & 3574 & 83 & 3657 & 0,295 & 886086,520 & 0,141 \\
\hline Martin_Sellner & $\begin{array}{l}\text { Alternativme- } \\
\text { dien Typ I, II }\end{array}$ & 3487 & 820 & 4307 & 0,346 & 18969719,602 & 0,140 \\
\hline Einzelfallinfos & $\begin{array}{l}\text { Alternativme- } \\
\text { dien Typ I, II }\end{array}$ & 3384 & 0 & 3384 & 0,000 & 0,000 & 0,130 \\
\hline IBDeutschland & $\begin{array}{l}\text { Alternativme- } \\
\text { dien Typ I, II }\end{array}$ & 3334 & 47 & 3381 & 0,286 & 536501,780 & 0,127 \\
\hline de_sputnik & $\begin{array}{l}\text { Alternativme- } \\
\text { dien Typ I, II }\end{array}$ & 2715 & 59 & 2774 & 0,304 & 1388523,747 & 0,094 \\
\hline Zeitgeschehen_ & $\begin{array}{l}\text { Alternativme- } \\
\text { dien Typ I, II }\end{array}$ & 2619 & 2555 & 5174 & 0,372 & 35392574,386 & 0,127 \\
\hline DanieleGanser & $\begin{array}{l}\text { Alternativme- } \\
\text { dien Typ I, II }\end{array}$ & 2568 & 2 & 2570 & 0,249 & 443199,077 & 0,077 \\
\hline TeamKenFM & $\begin{array}{l}\text { Alternativme- } \\
\text { dien Typ I, II }\end{array}$ & 2565 & 170 & 2735 & 0,329 & 6988721,507 & 0,079 \\
\hline Netzdenunziant & $\begin{array}{l}\text { Alternativme- } \\
\text { dien Typ I, II }\end{array}$ & 2481 & 2020 & 4501 & 0,354 & 6415490,078 & 0,118 \\
\hline unzensuriert & $\begin{array}{l}\text { Alternativme- } \\
\text { dien Typ I, II }\end{array}$ & 1129 & 69 & 1198 & 0,301 & 201652,689 & 0,052 \\
\hline
\end{tabular}

